\title{
Efficacy of Oral Supplementation with Silymarin and S-Adenosyl-L-Methionine in Patients with Non Alcoholic Fatty Liver Disease - A Pilot Study
}

\author{
Ilaria Giangrandi ${ }^{1,2}$, Monica Dinu ${ }^{1,2^{*}}$, Giuditta Pagliai ${ }^{1,2}$, Francesco Sofi $^{1,2,3}$ and Alessandro Casini ${ }^{1,2}$ \\ ${ }^{1}$ Department of Experimental and Clinical Medicine, University of Florence, Italy \\ ${ }^{2}$ Unit of Clinical Nutrition, University Hospital of Careggi, Florence, Italy \\ ${ }^{3}$ Don Carlo Gnocchi Foundation Italy, Onlus IRCCS, Florence, Italy
}

*Corresponding author: Monica Dinu, Department of Experimental and Clinical Medicine, University of Florence, Largo Brambilla 3 , Florence-50134, Italy, Tel: +39 055 7949420; Fax: +39 055 7949418; E-mail: mdinu@unifi.it

Received date: October 06, 2016, Accepted date: October 26, 2016, Publication date: October 31, 2016

Copyright: (c) 2016 Giangrandi I, et al. This is an open-access article distributed under the terms of the Creative Commons Attribution License, which permits unrestricted use, distribution, and reproduction in any medium, provided the original author and source are credited.

\begin{abstract}
Background and Aim: Non-alcoholic fatty liver disease (NAFLD) is the most common liver disease worldwide, affecting up to one-third of the population in industrialized countries. Silymarin and S-Adenosyl-L-Methionine (SAMe) have therapeutic potential for treatment of liver disease. The aim of the present pilot study was to evaluate the possible effect of a supplementation with Silymarin and SAMe in NAFLD patients.
\end{abstract}

Methods: Fifteen NAFLD patients (mean age: $46.3 \pm 15$ years) confirmed by ultra-sonographic findings were subjected to a 12-month oral supplementation with Silymarin and SAMe. Anthropometric measurements, biochemical parameters, liver function and hepatic fat content were assessed at baseline, at 6 months and at the end of treatment.

Results: After a 12-months' oral supplementation a significant improvement $(p<0.05)$ in several biochemical parameters such as total cholesterol (mean reduction: $-8.67 \mathrm{mg} / \mathrm{dL}$ ), insulin $(-1.34 \mathrm{U} / \mathrm{L})$ and HOMA-IR $(-1.35)$ was observed. Similarly, serum levels of alanine aminotransferase (ALT) and alkaline phosphatase (ALP) significantly decreased by $13 \%(-3.87 \mathrm{U} / \mathrm{L}$ and $-10.4 \mathrm{U} / \mathrm{L}$ respectively), with a small but significant amelioration in the risk profile. Finally, the ultrasonography examination revealed an improvement in liver echo-texture and a regression of the degree of hepatic steatosis, associated with a $22 \%$ increase of the Doppler Perfusion Index (DPI), which corresponds to better liver hemodynamic.

Conclusion: Our findings suggest that a prolonged Silymarin and SAMe supplementation could be used as an adjunctive therapy to improve metabolic risk profile and hepatic steatosis in patients with NAFLD. Further research is needed to confirm these preliminary results.

Keywords: Silymarin; S-adenosyl-L-methionine; NAFLD

\section{Introduction}

Non-alcoholic fatty liver disease (NAFLD) is the most common liver disease in the industrialized countries [1]. Its prevalence increased from less than $10 \%$ in the 1980 s, through $10 \%$ to $20 \%$ in the 1990 s, to current rates of $15 \%$ to $30 \%$ or higher [1]. NAFLD represents the outcome of genetically-determined interactions between a changing environment and a susceptible host. The environmental factors include an excess of energy intake, particularly in the form of cheap, highly processed simple carbohydrates and saturated fats, and reduced levels of physical fitness resulting from a sedentary lifestyle [2]. NAFLD embraces a pathological spectrum of liver disease, from cases of steatosis with virtually no evidence of hepatocellular injury or liver inflammation, often referred to as simple steatosis through steatohepatitis (NASH), to cases with cirrhosis [3]. Individuals with only hepatic steatosis (simple steatosis) infrequently show signs of any histologic progression, and are not at significant long-term risk of liver-related death [4], but those with advanced hepatic fibrosis are likely to experience liver-related complications (ascites, variceal bleeding, and/or hepatocellular carcinoma) [5].
Furthermore, hepatic necroinflammatory activity in patients with NAFLD is able to increase the risk of future cardiovascular disease [6], and confers a higher risk of unfavorable liver-related outcomes by promoting development of hepatic fibrosis. The lipid molecules that accumulate, together with triglycerides, in some NAFLD livers can participate directly in pathogenesis of the necroinflammatory element of NASH [7]. From the scientific literature it is known that high intracellular levels of Silymarin and S-Adenosyl-L-Methionine (SAMe) act in: reducing the uptake of exogenous toxic substances, increasing membrane fluidity, detoxifying the hepatic cell, preventing the depletion of glutathione and thereby increasing its availability, having anti-inflammatory activity and in promoting the regeneration of liver cells $[8,9]$. The aim of the present intervention study was to evaluate the possible effect of an oral supplementation of Silymarin and SAMe in NAFLD patients.

\section{Material and Methods}

\section{Study population}

Fifteen patients with a diagnosis of NAFLD referred to the Unit of Clinical Nutrition of the University Hospital of Careggi, Florence, Italy, 
and characterized by ultrasonography features indicative of NAFLD were eligible for enrolment. Written informed consent was obtained from all patients. The study protocol followed the ethical guidelines of the Declaration of Helsinki and was approved by our departmental Ethics Committee.

The inclusion criteria were as follows: age $>18$ years, lack of excessive alcohol consumption $(<20 \mathrm{~g} / \mathrm{d})$, ultrasound examination demonstrating fatty liver, negative diagnostic tests for viral hepatitis and absence of other causes of liver disease. Exclusion criteria included a history of alcohol intake $>20 \mathrm{~g} / \mathrm{d}$, use of drugs known to be associated with liver steatosis, undernutrition, chronic viral hepatitis and chronic liver disease of other causes (autoimmune hepatitis, primary biliary cirrhosis, primary sclerosing cholangitis, hereditary haemochromatosis, Wilson's disease, a1-anti-trypsin deficiency, coeliac disease). Pregnant and breastfeeding women were also excluded.

\section{Study design}

All patients were subjected to a 12-months' oral supplementation with 1 tablet per day of Duoliver (Biocure Srl, Milan), a food supplement containing Silymarin (140 mg) and SAMe $(200 \mathrm{mg})$ without dietary guidelines or other lifestyle changes. The products were packed in labelled boxes. Compliance was verified by counting the empty boxes on return. A medical examination with measurement of body mass index (BMI), blood tests and liver ultrasonography was performed at baseline, at 6 months and at the end of the treatment. BMI was calculated as weight $(\mathrm{kg}) /$ height $\left(\mathrm{m}^{2}\right)$.

The laboratory evaluation included total cholesterol, LDLcholesterol, HDL-cholesterol, triglycerides, fasting glucose blood levels, insulin blood levels, insulin resistance index (HOMA-IR), aspartate aminotransferase (AST), alanine aminotransferase (ALT), gamma glutamyl transferase ( $\gamma$-GT), alkaline phosphatase (ALP), and total and fractionated bilirubin. All subjects were interviewed by means of an ad hoc questionnaire for the frequency and quantity of recreational activities performed during the preceding year. Hence, physical activity was graded into qualitative terms such as absent or light (i.e. inactive or either occasional walking or recreational activity only), moderate (i.e. frequent recreational activities, regular walking for 30 minutes three to five times per week or sporting exercise at least once a week) and intense (i.e. sporting activity at least twice a week, plus frequent recreational activities or walking, or frequent sporting activities only). The dietary profile of the study patients was evaluated by an experienced clinical dietician with the aid of software specific for the analysis of food habits and the estimation of nutrient and caloric intake.

\section{Fatty liver evaluation}

B-mode ultrasound and duplex Doppler investigation were performed by the same expert operator using a high-quality ultrasound device equipped with a $5 \mathrm{MHz}$ Convex array transducer (Voluson 530 DMT; Kretz Technik AG, Zipf, Austria). The patients fasted for at least $12 \mathrm{~h}$ and underwent scanning in a supine position; data were obtained during suspended inspiration. In a B-mode imaging evaluation, the liver echo-texture was scored on a four-grade scale, by comparing it with the right kidney cortical echogenicity: Grade 0, absent steatosis; Grade 1, mild steatosis (lightly and homogeneously increased liver echo-texture, with patent intrahepatic vascular pattern; absent posterior attenuation); Grade 2, moderate steatosis (moderate increase of liver echo-texture; partial dimming of the vessels; early posterior attenuation); and Grade 3, severe steatosis (diffuse increase of liver echogenicity, with no longer visible intrahepatic vessels; heavy posterior attenuation). Duplex Doppler ultrasound allowed a semi-quantitative measurement of fatty storage on the basis of the Doppler Perfusion Index (DPI) (i.e. the ratio between hepatic artery blood flow and total liver blood flow).

\section{Laboratory parameters}

Venous blood samples anticoagulated with $0.129 \mathrm{M}$ sodium citrate (ratio 9:1) were collected from the antecubital vein into evacuated plastic tubes (Vacutainer; BD, Milan, Italy), after overnight fasting. Whole venous blood was also collected in tubes without anticoagulant. Citrated and serum samples were centrifuged at $2,000 \times \mathrm{g}$ for $10 \mathrm{~min}$ at $4^{\circ} \mathrm{C}$, and supernatants were stored in aliquots at $-80^{\circ} \mathrm{C}$ until assays. Lipid variables, blood glucose, insulin, and hepatic function tests were assessed by conventional methods, as well as the HOMA-IR.

\section{Statistical analysis}

Statistical analysis was performed using the SPSS (Statistical Package for Social Sciences, Chicago, IL, USA) software for Macintosh (Version 20.0). Results were expressed as means \pm standard deviation. The Chi-square test was used to identify statistically significant differences between categorical variables. The Wilcoxon test for related data was used to evaluate differences between two time-points. A general linear model for repeated measurements, with adjustments for age, sex and BMI change, was used to compare differences between baseline and the end of the intervention period. A P value $\leq 0.05$ was considered significant for all the analyses.

\section{Results}

Between January, 2015 and May, 2015 a total of 15 patients (11 females, 4 males; mean age: $46.3 \pm 15$ years) with NAFLD were included in the protocol. Participants received a Silymarin plus SAMe supplement for 12 months. None of them withdrew from the study during the research period and no adverse events were observed. At baseline, the average BMI was $30.9 \pm 8.9 \mathrm{~kg} / \mathrm{m}^{2}$, with 7 (47\%) patients categorized as obese or extremely obese and $4(27 \%)$ patients classified as overweight. The assessment of liver steatosis showed a moderate/ severe steatosis in $6(40 \%)$ patients.

After 6 and 12 months of food supplementation with Silymarin and SAMe, BMI did not change significantly with respect to baseline $(p=0.65)$. In order to analyse the changes in the biochemical variables after the intervention, a general linear model adjusted for age, sex and BMI change was performed. As reported in Table 1, supplementation was found to result in a significant reduction by $4 \%$ (mean reduction: $-8.67 \mathrm{mg} / \mathrm{dL} ; \mathrm{p}=0.01$ ) for total cholesterol levels with respect to baseline. Also insulin levels and HOMA-IR decreased significantly by $9 \%$ and $15 \%$, respectively $(-1.34 \mathrm{U} / \mathrm{L} ; \mathrm{p}<0.01$ and $-1.35 ; \mathrm{p}=0.03)$ with an amelioration of the metabolic status of patients. Non-significant differences were observed for LDL-cholesterol, HDL-cholesterol and triglycerides.

With regard to the liver function tests, at the end of the study we observed a significant reduction by $13 \%$ in the levels of both ALT $(-3.87 \mathrm{U} / \mathrm{L} ; \mathrm{p}=0.01)$ and ALP $(-10.4 \mathrm{U} / \mathrm{L} ; \mathrm{p}=0.04)$, with non-significant changes in the other parameters (Table 2). The echo-Doppler examination revealed a $22 \%$ increase in the hepatic DPI, moving from a mean of $0.18 \pm 0.07$ at baseline to $0.22 \pm 0.05$ at the end of the treatment (normal value range: $0.20-0.30$ ), although this was not 
Citation: Giangrandi I, Dinu M, Pagliai G, Sofi F, Casini A (2016) Efficacy of Oral Supplementation with Silymarin and S-Adenosyl-L-Methionine in Patients with Non Alcoholic Fatty Liver Disease - A Pilot Study. Altern Integr Med 5: 224. doi:10.4172/2327-5162.1000224

Page 3 of 5

significant $(\mathrm{p}=0.08)$. However, ultrasonography indicated a regression of steatosis in a consistent proportion of patients: after a 12-months oral supplementation with Silymarin and SAMe, 5 patient out of 6 with moderate/severe steatosis regressed to mild steatosis (Figure 1).

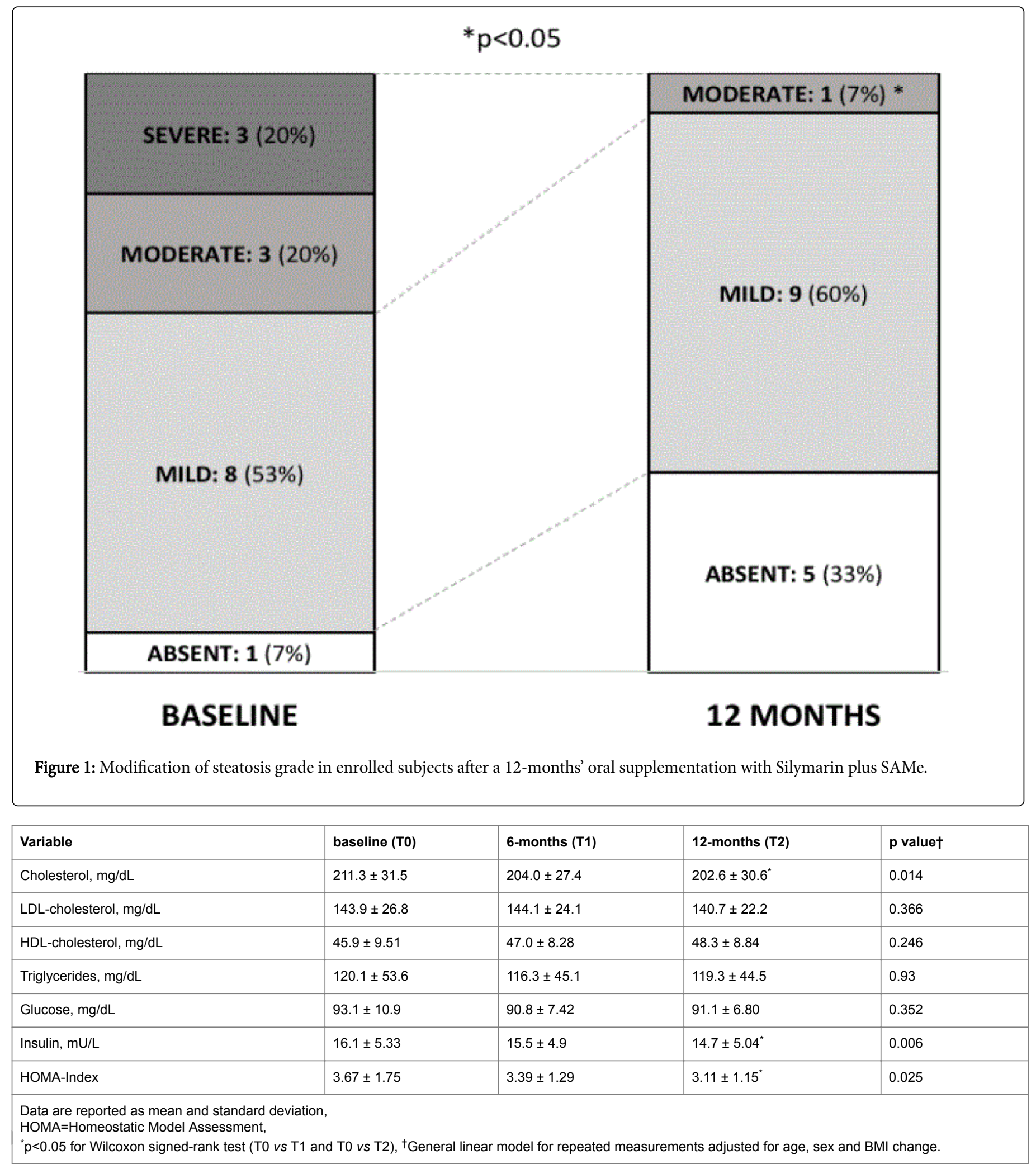

Table 1: Modification of biochemical parameters in enrolled subjects after 6- and 12-months' oral supplementation with Silymarin plus SAMe. 
Page 4 of 5

\begin{tabular}{|l|l|l|l|l|}
\hline Variable & baseline (T0) & $\mathbf{6 - m o n t h s ~ ( T 1 ) ~}$ & 12-months (T2) & p value \\
\hline AST, U/L & $21.9 \pm 7.30$ & $21.3 \pm 7.07$ & $21.2 \pm 6.71$ & 0.443 \\
\hline ALT, U/L & $30.0 \pm 15.4$ & $28.6 \pm 13.3$ & $26.1 \pm 13.5^{*}$ & 0.013 \\
\hline Y-GT, U/L & $44.4 \pm 39.9$ & $45.7 \pm 60.1$ & $44.6 \pm 59.1$ & 0.974 \\
\hline ALP, U/L & $80.7 \pm 34.3$ & $77.5 \pm 31.6$ & $70.3 \pm 30.7^{*}$ & 0.039 \\
\hline Total bilirubin, $\mathrm{mg} / \mathrm{dL}$ & $0.75 \pm 0.23$ & $0.70 \pm 0.20$ & $0.81 \pm 0.16$ & 0.235 \\
\hline Direct bilirubin, $\mathrm{mg} / \mathrm{dL}$ & $0.18 \pm 0.08$ & $0.21 \pm 0.10$ & $0.57 \pm 0.16$ & 0.097 \\
\hline Indirect bilirubin, $\mathrm{mg} / \mathrm{dL}$ & $0.57 \pm 0.22$ & $0.49 \pm 0.22$ & 0.875 \\
\hline
\end{tabular}

Data are reported as mean and standard deviation,

AST: Aspartate Aminotransferase; ALT: Alanine Aminotransferase; y-GT: Gamma Glutamyl Transferase; ALP: Alkaline Phosphatase,

* $\mathrm{p}<0.05$ for Wilcoxon signed-rank test (T0 vs T1 and T0 vs T2),

${ }^{\dagger}$ General linear model for repeated measurements adjusted for age, sex and BMI change.

Table 2: Modification of hepatic function tests in enrolled subjects after 6- and 12-months' oral supplementation with Silymarin plus SAMe.

\section{Discussion}

The current study reported a beneficial effect of an oral supplementation on the metabolic risk profile in patients with NAFLD. Indeed, after a 12-month oral supplementation, the ultrasonography examination revealed an improvement in liver echo-texture and a regression of the degree of hepatic steatosis, associated with a remarkable increase of DPI, which corresponds to better liver hemodynamic. Moreover, a significant improvement in several biochemical parameters such as total cholesterol, insulin, HOMA-IR, ALT and ALP was also observed after the intervention period.

To date, lifestyle modifications, healthy diet and physical activity are the most effective treatment for NAFLD, but for many people they are difficult to achieve and maintain [10]. Therefore, over the past years, several therapeutic proposals such as weight reducing, lipid-lowering, and insulin-sensitizing drugs have been proposed. These agents should act to reduce the link between adipose tissue and liver function by acting as anti-inflammatory and immunomodulatory agents. However, a validated and unique approach has not yet been obtained [10]. On the other hand, the use of complementary medicines, such as natural antioxidants, may have therapeutic potential. In fact, oxidative stress is thought to play a central role in the aetiology of NAFLD, as fatty acid oxidation produces reactive oxygen species, which cause direct cellular damage and activate pro-inflammatory cytokines. (1) Derived from the milk thistle plant (Silybum marianum), Silymarin is an example of a modern drug that arose from traditional medicine practices. The hepatic protection of this flavonoid complex has been attributed to its antioxidant, anti-inflammatory and antifibrotic properties [11].

This study is one of the few clinical trials that assessed the potential role of Silymarin and SAMe in the treatment of NAFLD. Supplementation with Silymarin alone has been investigated in two Iranian studies [12,13], exhibiting large effects on serum levels of ALT and AST. In parallel, an initial pilot study [14] and a subsequent large randomised placebo-control trial [15], conducted by Loguercio et al. [15], reported that a supplementation with Silybin (the major active constituent of Silymarin) combined with vitamin $\mathrm{E}$ and phosphatidylcholine improved insulin resistance and liver histology in patients with NAFLD. In a further clinical trial, food supplement containing vitamin E, L-glutathione, L-cysteine, L-methionine and Silybum marianum, given twice a day for 3 months in 72 subjects with NAFLD, reduced the biochemical, inflammatory and ultrasonic indices of hepatic steatosis [16]. Similar results were obtained in three studies [17-19] where the addition of Silymarin improved the efficacy of the lifestyle intervention. Conversely, Sorrentino et al. [20] reported a significant improvement of biometric parameters and liver steatosis, but not a reduction of liver function tests after supplementation with Silymarin. Finally, one study [21] comparing disposition of silymarin between NAFLD and Hepatitis C (HCV) patients has found that the effects of silymarin on liver disease progression was greater in NAFLD patients than in HCV patients.

While the positive effect of Silymarin on biochemical parameters and liver function has been tested in several clinical trials, there are no human studies that currently address the effect of SAMe in NAFLD patients. Indeed, the evidence of a role for SAMe in the pathogenesis of NAFLD has mainly been based on the study of animal models. Current knowledge suggests that altered hepatic SAMe level may lead to NAFLD development as SAMe is a methyl donor in the synthesis of phosphatidylcholine, which is required for VLDL assembly and hepatic triglyceride export [20]. In addition, reduced SAMe levels seem to sensitize the liver to lipopolysaccharide-induced injury and promotes expression and release of pro-inflammatory cytokines [22].

Although results are promising, the number of participants represents a limitation. Further and larger studies are needed before drawing any firm conclusions. In addition, up to now, there is no evidence on the ideal quantity and duration of supplementation with Silymarin plus SAMe for NAFLD. We cannot exclude that a higher 
dosage and/or a prolonged intake could reach even better outcomes. A current problem of clinical trials conducted on NAFLD patients is how to measure the outcome [23]. In particular, routine laboratory tests are helpful but not sufficient to assay the effectiveness of treatment, while needle liver biopsy, that provides the most conclusive evaluation both for diagnosis and follow-up, cannot be carried out in all patients because of invasiveness and possible complications. In the present study, in order to improve the accuracy of the evaluation, besides the liver function tests we performed ultrasonography to assess the hepatic fat content, and duplex doppler ultrasound, which provides a quantitative, non-invasive evaluation of NAFLD by measuring hepatic artery and portal vein blood flow.

In conclusion, our findings suggest that a prolonged Silymarin plus SAMe oral supplementation might have positive effects in patients with NAFLD, improving hepatic steatosis and insulin resistance, and reducing cholesterol and liver enzymes level. In patients who fail to change their lifestyle, Silymarin and SAMe could be a valid alternative therapeutic option, where appropriate as a complementary treatment associated with other therapeutic programs. Further research is needed to confirm these preliminary results.

\section{Acknowledgments}

This work was supported in part by a grant from Biocure S.r.l., Milan, Italy.

\section{References}

1. Rinella ME (2015) Nonalcoholic fatty liver disease: a systematic review. JAMA 313: 2263-2273.

2. Thoma C, Day CP, Trenell MI (2012) Lifestyle interventions for the treatment of non-alcoholic fatty liver disease in adults: a systematic review. J Hepatol 56: 255-266.

3. Kleiner DE, Makhlouf HR (2016) Histology of Nonalcoholic Fatty Liver Disease and Nonalcoholic Steatohepatitis in Adults and Children. Clin Liver Dis 20: 293-312.

4. Dam-Larsen S, Franzmann M, Andersen IB, Christoffersen P, Jensen LB, et al. (2004) Long term prognosis of fatty liver: risk of chronic liver disease and death. Gut 53: 750-755.

5. Bhala N, Angulo P, van der Poorten D, Lee E, Hui JM, et al. (2011) The natural history of nonalcoholic fatty liver disease with advanced fibrosis or cirrhosis: an international collaborative study. Hepatology 54: 1208-1216.

6. Motamed N, Rabiee B, Poustchi H, Dehestani B, Hemasi GR, et al. (2016) Non-alcoholic fatty liver disease (NAFLD) and 10-year risk of cardiovascular diseases. Clin Res Hepatol Gastroenterol.

7. Goh GB, McCullough AJ (2016) Natural History of Nonalcoholic Fatty Liver Disease. Dig Dis Sci 61: 1226-1233.

8. Abenavoli L, Aviello G, Capasso R, Milic N, Capasso F (2011) Milk thistle treatment of nonalcoholic fatty liver disease. Hepat Mon 11: 173-177.
9. Anstee QM, Day CP (2012) S-adenosylmethionine (SAMe) therapy in liver disease: a review of current evidence and clinical utility. J Hepatol 57: 1097-1109.

10. Scherer A, Dufour JF (2016) Treatment of Non-Alcoholic Fatty Liver Disease. Dig Dis 34: 27-31.

11. Parmar M, Gandhi T (2008) Hepatoprotective herbal drug, silymarin from experimental pharmacology to clinical medicine-A review. Pharmacogn Rev 2: 102.

12. Hajaghamohammadi AA, Ziaee A, Rafiei R (2008) The Efficacy of Silymarin in Decreasing Transaminase Activities in Non-Alcoholic Fatty Liver Disease: A Randomized Controlled Clinical Trial. Hepat Mon 8: 191-195.

13. Hajiaghamohammadi AA, Ziaee A, Oveisi S, Masroor H (2012) Effects of metformin, pioglitazone, and silymarin treatment on non-alcoholic Fatty liver disease: a randomized controlled pilot study. Hepat Mon 12: e6099.

14. Loguercio C, Federico A, Trappoliere M, Tuccillo C, de Sio I, et al. (2007) The effect of a silybin-vitamin e-phospholipid complex on nonalcoholic fatty liver disease: a pilot study. Dig Dis Sci 52: 2387-2395.

15. Loguercio C, Andreone P, Brisc C, Brisc MC, Bugianesi E, et al. (2012) Silybin combined with phosphatidylcholine and vitamin $\mathrm{E}$ in patients with nonalcoholic fatty liver disease: a randomized controlled trial. Free Radic Biol Med 52: 1658-1665.

16. Cacciapuoti F, Scognamiglio A, Palumbo R, Forte R, Cacciapuoti F (2013) Silymarin in non alcoholic fatty liver disease. World J Hepatol 5: 109-113.

17. Aller R, Izaola O, Gómez S, Tafur C, González G, et al. (2015) Effect of silymarin plus vitamin $\mathrm{E}$ in patients with non-alcoholic fatty liver disease. A randomized clinical pilot study. Eur Rev Med Pharmacol Sci 19: 3118-3124.

18. Hashemi SJ, Hajiani E, Sardabi EH (2009) A placebo-controlled trial of silymarin in patients with nonalcoholic fatty liver disease. Hepat Mon 9: 265-270.

19. Abenavoli L, Greco M, Nazionale I, Peta V, Milic N, et al. (2015) Effects of Mediterranean diet supplemented with silybin-vitamin E-phospholipid complex in overweight patients with non-alcoholic fatty liver disease. Expert Rev Gastroenterol Hepatol 9: 519-527.

20. Sorrentino G, Crispino P, Coppola D, De Stefano G (2015) Efficacy of lifestyle changes in subjects with non-alcoholic liver steatosis and metabolic syndrome may be improved with an antioxidant nutraceutical: a controlled clinical study. Drugs R D 15: 21-25.

21. Schrieber SJ, Hawke RL, Wen Z, Smith PC, Reddy KR, et al. (2011) Differences in the disposition of silymarin between patients with nonalcoholic fatty liver disease and chronic hepatitis C. Drug Metab Dispos 39: 2182-2190.

22. Chawla RK, Bonkovsky HL, Galambos JT (1990) Biochemistry and pharmacology of S-adenosyl-L-methionine and rationale for its use in liver disease. Drugs 40: 98-110.

23. Sumida Y, Nakajima A, Itoh Y (2014) Limitations of liver biopsy and noninvasive diagnostic tests for the diagnosis of non-alcoholic fatty liver disease/non-alcoholic steatohepatitis. World J Gastroenterol 20: 475-485. 